$43(1) \mid 2014$

Varia

Nanterre (France), 21-22 marzo 2014

\title{
Séminaire «Patrimoine(s) en Équateur : politiques culturelles et politiques de conservation»
}

David Macias, Alexis Medina et Emmanuelle Sinardet

\section{OpenEdition}

Journals

Édition électronique

URL : http://journals.openedition.org/bifea/4419

DOI : 10.4000/bifea.4419

ISSN : 2076-5827

Éditeur

Institut Français d'Études Andines

Édition imprimée

Date de publication : 1 mai 2014

Pagination : 181-186

ISSN : 0303-7495

Référence électronique

David Macias, Alexis Medina et Emmanuelle Sinardet, « Séminaire «Patrimoine(s) en Équateur : politiques culturelles et politiques de conservation» », Bulletin de l'Institut français d'études andines [En ligne], 43 (1) | 2014, mis en ligne le 08 mai 2014, consulté le 07 novembre 2020. URL : http:// journals.openedition.org/bifea/4419; DOI : https://doi.org/10.4000/bifea.4419

\section{(c) $($ ) $\odot$}

Les contenus du Bulletin de l'Institut français d'études andines sont mis à disposition selon les termes de la licence Creative Commons Attribution - Pas d'Utilisation Commerciale - Pas de Modification 4.0 International. 


\section{SÉMINAIRE «PATRIMOINE(S) EN ÉQUATEUR : POLITIQUES CULTURELLES ET POLITIQUES DE CONSERVATION»}

Nanterre (France), 21-22 marzo 2014

Les 21 et 22 mars, sur le campus de Nanterre, s'est tenu le colloque international «Patrimoine(s) en Équateur : politiques culturelles et politiques de conservation », organisé par le Centre d'études équatoriennes de l'Université Paris Ouest Nanterre - La Défense. Ce colloque a reçu l'appui de plusieurs entités de Paris Ouest : du CRIIA (Centre de recherche ibérique et ibéro-américaine) relevant de l'Équipe d'Accueil 369, de l'École doctorale Lettres, langues, Spectacles (ED 138), et de I'UFR LCE de I'Université Paris Ouest. II est le fruit d'une collaboration de longue date, puisqu'elle remonte à la création du Centre d'études équatoriennes en 1972, avec l'Ambassade de l'Équateur en France et la Délégation de l'Équateur auprès de I'Unesco. Et il n'aurait pu se tenir sans l'aide précieuse de I'Institut français d'études andines (IFEA).

Jean Jacques Courtet, descendant de Juan Montalvo, a fait don à l'Etat équatorien de la fûte et d'une photographie qui ont appartenu à Juan Montalvo". Cette remise a eu lieu au début du colloque.

Pas moins de 25 communications y ont été présentées par des chercheurs venus de différentes Universités et Centres de recherche en France, Allemagne, Cuba et Équateur, mais aussi par les professionnels des musées et institutions culturelles équatoriennes. Privilégiant une approche pluridisciplinaire, le colloque a permis de faire dialoguer la réflexion et les points de vue d'historiens, géographes, linguistiques, sociologues, anthropologues, chercheurs en littérature, historiens de l'art et conservateurs. Les débats qui ont suivi chaque séance se sont avérés à ce titre des espaces riches d'échanges.

La réflexion a porté sur la relation au(x) patrimoine(s) en Équateur, fil rouge de ces deux journées denses et fructueuses, alors que la nouvelle Constitution de 2008 en fait un enjeu clé des politiques culturelles, en élargissant la définition même de la notion de patrimoine pour l'articuler au Sumak Kawsay ou « Buen Vivir » (« Bien 
vivre »), concept politique au cœur du nouveau projet de construction nationale. L'Équateur n'est pas le seul pays à reconnaître de nouvelles acceptions de la notion de patrimoine, patrimoines immatériels, patrimoines naturels, par exemple ; mais il place le patrimoine au cœur d'un projet qui dépasse le seul cadre des politiques de conservation et des politiques culturelles, dont I'initiative originale Yasuní-ITT marque d'ailleurs un tournant.

Les participants au colloque se sont efforcés, à la lumière d'exemples concrets et d'études de cas, d'apprécier ces évolutions récentes, tout en les replaçant dans une perspective historique qui permette aussi de cerner les modalités des redéfinitions d'une culture nationale considérée comme genuinamente équatorienne. Ils ont pu ainsi observer les différents processus de patrimonialisation à l'œuvre en Équateur depuis le 19e siècle, à même de rendre compte de la transformation et de l'élargissement des politiques culturelles, qu'illustrent notamment les politiques urbaines de restauration des centres historiques de la fin du $20^{\mathrm{e}}$ siècle et la multiplication et promotion des parcs nationaux au $21^{\mathrm{e}}$ siècle. Ils ont également observé le rôle des institutions publiques, mais aussi celui qui échoit aux citoyens - et que ces derniers s'approprient, le cas échant, pour revitaliser des éléments culturels folklorisés, négligés ou en passe de tomber dans l'oubli.

Les interventions se sont organisées en 8 séances de travail qui portaient chacune sur les spécificités de la notion de patrimoine en Équateur, sur les outils des politiques de conservation ou encore sur les enjeux politiques et sociaux des processus de patrimonialisation à l'œuvre.

Le colloque a été ouvert par Monsieur Balaudé, Président de I'Université Paris Ouest, et par Monsieur Játiva, Ambassadeur de la République de l'Équateur à Paris. Madame Sinardet, directrice du Centre d'études équatoriennes, a adressé, au nom du Centre, ses remerciements aux partenaires qui ont permis la tenue de ces deux journées.

La première matinée de travail a été consacrée aux relations entre le(s) patrimoine(s) et les langues et littératures1. Une première séance a observé, sur la base d'un corpus littéraire, les tentatives pour fixer,

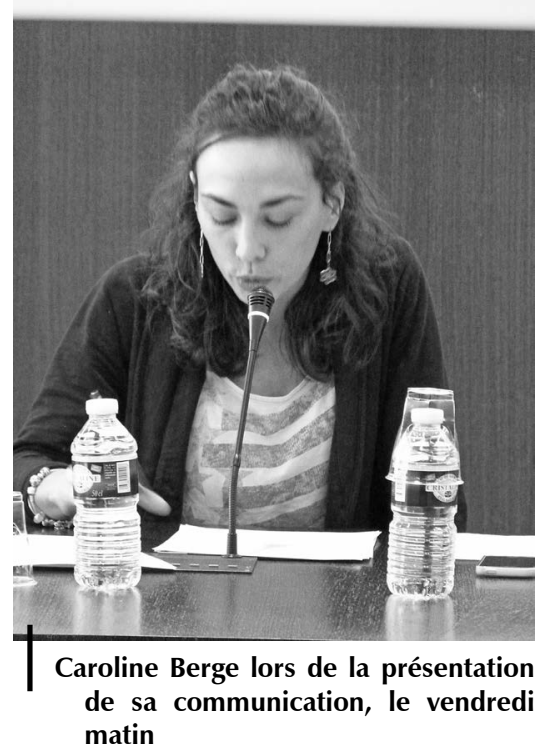

1 Caroline Berge (Université Paris Ouest), « Le langage dans la littérature équatorienne du XXe siècle : entre identité et dépendance culturelle vis-à-vis de I'Occident »; Rut Román (Universidad Laica Eloy Alfaro de Manabí), « La pluralidad textual de las literaturas de Manabí » ; David Macías Barres (Université Paris Ouest), Patrimonio lingüístico e identidad cultural : el montubio y el amorfino » ; Marianela Rosa Peña Lora (Casa del Caribe), "Una metodología para la salvaguarda del patrimonio inmaterial de la cuentería popular" ; Marleen Haboud (Pontificia Universidad Católica del Ecuador, Quito), « Rencuentro y preservación del patrimonio lingüístico y cultural del Ecuador : el rol de la documentación activa ». 
préserver et transmettre une langue nationale équatorienne, mais aussi un parler local dans le cas montubio. Ces communications mettent en évidence les enjeux identitaires des processus de patrimonialisation tant dans le cas d'une langue dite savante que - plus récemment — dans le cas de parlers populaires. Patrimoine et langage entrent alors en interaction pour proposer une lecture des origines, objet d'appropriation comme support stable pour une identité collective. En témoignent la première communication sur la quête d'une langue nationale propre à la littérature du $20^{\mathrm{e}}$ siècle, la seconde sur les littératures du Manabi comme « fixant » un parler spécifique et différenciateur, puis la troisième consacrée à l'amorfino, genre à part entière, il y a peu considéré comme une forme poétique populaire de second ordre, mais qu'une récente réforme éducative fait désormais figurer dans les programmes scolaires comme élément du patrimoine culturel national. La seconde séance s'est penchée sur les outils de conservation utilisés en Équateur pour préserver le patrimoine immatériel linguistique, notamment les parlers et les contes populaires, et elle revient sur deux initiatives originales des politiques culturelles menées récemment : une vaste enquête sociolinguistique géo-référencée en 2009 menée dans toutes les régions du pays, d'une part, une méthodologie de conservation des contes oraux des communautés indiennes andines, d'autre part, dans le cadre d'une politique de préservation plus vaste des savoirs dits ancestraux et traditionnels, aujourd'hui pleinement reconnus comme des éléments du patrimoine national. Ramiro Noriega, Ministre et représentant du Service culturel de l'Ambassade de l'Équateur, a exposé les enjeux des nouvelles politiques culturelles du gouvernement Correa dans la conservation de ce vaste patrimoine et le rôle du Ministère de la Culture.

Cette nouvelle politique de conservation du patrimoine immatériel que représentent les savoirs ancestraux - fragilisés et parce que fragilisés — a été au cœur des deux séances de l'après-midi. Directement ou indirectement, les six communications se sont penchées sur la notion de Sumak Kawsay (« Bien Vivre ») et l'impact de sa reconnaissance par la Constitution de 2008. Elles sont ainsi revenues sur les initiatives récentes en faveur de la conservation d'un patrimoine naturel désormais défini en termes éminemment identitaires, non seulement par les cosmovisions autochtones mais par la Constitution même². Les communications pointent toutes une grande adaptation des communautés indiennes, qui s'approprient les nouveaux outils de conservation et les instruments

2 Philipp Altmann (FU, Berlin), «El Sumak Kawsay y el Patrimonio Ecuatoriano »; Ana Gendron (IHEAL, Université Paris 3), «Parole Mythique, rituels et développement des communautés indiennes »; Julie Carpentier (Université Paris-Ouest/IFEA), « Somos shuar y debemos identificarnos como shuar ». Les enjeux identitaires et politiques de la mise en tourisme du patrimoine local dans la communauté de Chico-Méndez ; Christine Récalt (Université Montpellier 3/IRD), « L'eau, un patrimoine en discussion entre l'État équatorien et les communautés autochtones» ; Ibtissem Ben Dridi (EHESS), « La montagne en héritage. Construction d'une fierté identitaire au nord de la Province du Chimborazo »; Nicole Fourtané (Université de Lorraine), « Les populations autochtones de I'Amazonie face à la compagnie pétrolière Chevron-Texaco : un procès historique » ; Cecilia Miño Grijalva (Ministerio de Energía y Minas, Plan Ambiental del Ecuador), « La minería destruyó lo sagrado ». 
légaux et juridiques que ceux-ci mettent à leur disposition, pour promouvoir leur propre développement. Ces communications invitent à penser les processus de patrimonialisation comme les facteurs d'une possible dynamisation culturelle et économique - notamment via l'écotourisme - des communautés indiennes. Toutefois, ces démarches ne vont pas sans tensions avec les acteurs économiques locaux, publics ou privés, comme le soulignent les trois communications de la deuxième séance de l'après-midi. Celles-ci, par des études de cas, illustrent les nombreux conflits qui surgissent autour de l'exploitation des ressources naturelles — eau, minerais, pétrole — , quand ces ressources peuvent aussi être considérées comme les éléments d'un patrimoine naturel à préserver, en l'occurrence comme les éléments d'une Pachamama proclamée sujet de droit par la Constitution de 2008, un bouleversement majeur et inédit.

La deuxième journée de travail débute par une séance qui s'efforce d'établir les possibles relations entre la construction d'un imaginaire collectif national et les processus de patrimonialisation, lesquels sélectionnent des éléments jugés dignes de faire partie d'un patrimoine genuinamente équatorien pour mieux préserver une identité nationale en constante mutation3. Parmi ces éléments, un cas particulièrement intéressant est la consécration du Sacré Cœur de Jésus comme premier pas pour définir, au 19e siècle, durant la période du progressisme, les Équatoriens comme un peuple élu à l'instar du peuple d'Israël, un peuple appelé à devenir le fer de lance de la préservation d'un catholicisme pensé comme le fondement de l'identité nationale. Cette patrimonialisation du Sagrado Corazón de Jesús se produit au moment même où apparaissent les premiers signes d'une sécularisation certes timide, mais annonciatrice du grand bouleversement que sera la Révolution libérale et laïque de la fin du $19^{\mathrm{e}}$. Cette communication invite à penser le patrimoine comme l'instrument d'une stratégie idéologique à caractère conservateur, au service de la préservation d'un idéal d'une nation « pure » mais vécue comme menacée et fragile. La deuxième communication explore un élément au cœur même de l'imaginaire collectif de l'Équateur, pays qui tire son nom de sa position géographique sur la ligne de l'Équateur : l'élément équinoxial, paradoxalement très rarement étudié. Au prisme des études culturelles, sur la base d'un vaste corpus, notamment pictural et littéraire, la communication observe comment l'élément équinoxial est représenté puis progressivement patrimonialisé à travers les discours sur les héritages naturels. Elle cerne aussi les limites d'une telle démarche de patrimonialisation, en particulier le risque de la réduction qui privilégierait Pichincha au détriment du reste du pays dans la représentation de l'équatorianité. La troisième communication revient sur l'évolution d'un élément

3 Alexis Medina (Université Paris Ouest), "La patrimonialisation de la consécration de l'Équateur au Sacré Cœur de Jésus pendant la période progressiste (1883-1895)" ; Esteban Ponce Ortiz (Université laica Eloy Alfaro de Manabí), «Patrimonialización de lo Equinoccial : el problema de los equinoccial como condición imaginaria, potencialidades y riesgos »; Nasser Rebaï (Université Paris 1 PanthéonSorbonne), «Émigration paysanne et mutation du patrimoine dans les campagnes du Cañar et de l'Azuay ». 
essentiel du patrimoine national dans l'imaginaire collectif, traditionnellement associé pour tous les Équatoriens à la singulière beauté des Andes : les paysages agraires, éléments d'un patrimoine rural défini comme l'ensemble des attributs et des « géosymboles » qui caractérisent une campagne. Avec une émigration soutenue qui transforme profondément les activités agricoles, ces paysages traditionnels sont modifiés, comme l'analyse l'étude des cas des provinces

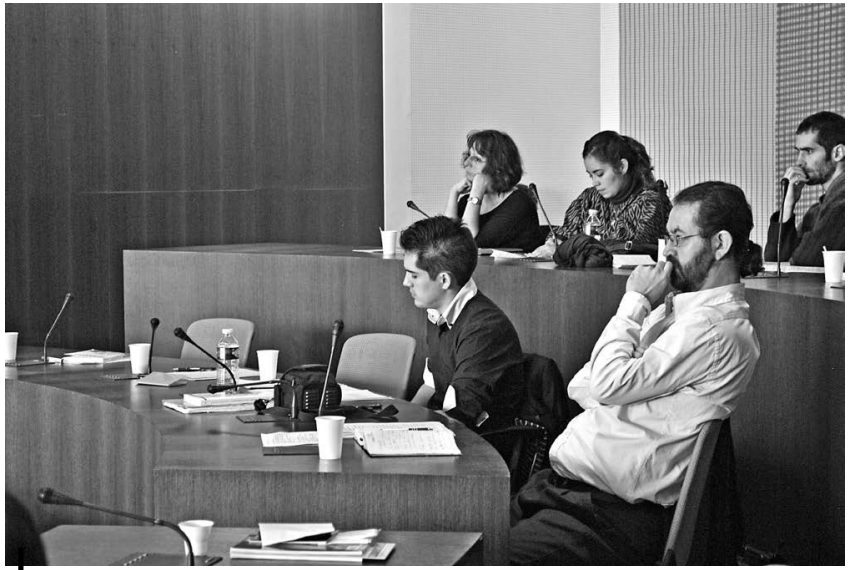

Anne-Claudine Moral (rang du haut, à droite); rang du bas: Alexis Medina à gauche, Esteban Once Ortiz à droite du Cañar et de l'Azuay, où la dynamique migratoire est la plus importante. L'étude observe alors les signes d'un 《 renouveau »du patrimoine rural, une reformulation que permettent certains outils de conservation; elle envisage également les stratégies politiques à mettre en œuvre pour le maintien de l'agriculture familiale dans la sierra, laquelle peut également être envisagée comme l'instrument de la sauvegarde d'une partie du patrimoine culturel des Andes équatoriennes.

Les deux séances suivantes, en fin de matinée et en début d'après-midi, se sont attachées aux réformes récentes des politiques culturelles patrimoniales, à travers l'étude de plusieurs initiatives représentatives 4 : la mise en place d'un réseau national des musées en Équateur, les interactions du musée avec la communauté dans lequel il s'implante pour favoriser l'appropriation par les citoyens les moins favorisés d'un patrimoine culturel savant - le cas du Musée Camilo Egas est à ce titre parlant - l'emploi de l'image animée en vue de la préservation et de la transmission des paysages culturels. Trois communications ont ensuite présenté les efforts pour la conservation et la mise en valeur du patrimoine urbain, à la lumière de trois cas emblématiques qui ont mobilisé les acteurs tant locaux que nationaux et qui ont abouti à une réappropriation par les citoyens de l'espace urbain,

4 María José Jarrín (Université Paris 1 Panthéon-Sorbonne), « Le patrimoine muséal équatorien : la mise en place d'un réseau national des musées »; Lorena Cisneros (Musée Camilo Egas, Quito), «La relación museo-comunidad : el caso del Museo Camilo Egas »; Mariluz Paredes Barragán (Senescyt), «L'usage de l'image animée comme moyen pour préserver les paysages culturels et le patrimoine intangible en Équateur»; Anne Collin Delavaud (IHEAL, Université Paris 3), « Des actions de restauration du centre de Quito à I'aménagement de la métropole » ; Lucía Durán et Eduardo Kingman (FLACSO-Ecuador), « Patrimonio cultural, renovación urbana y el problema de lo social »; Sandra Pacheco Fernández (Fundación Municipal El Barranco, Cuenca), « Cuenca Patrimonio Verde y Edificado : proyectos de revitalización urbana en el centro histórico » ; Diana Sarrade Cobos (Université de Bordeaux Montesquieu), «Projet Guayaquil Écologique : nouvelles perspectives pour la réhabilitation de I’Estero Salado ». 
dans le cadre d'ambitieux projets de « revitalisation » de quartiers populaires: la restauration et l'aménagement du centre historique de Quito, de celui de Cuenca ou encore de l'Estero Salado à Guayaquil. Une communication remet en perspective toutefois les succès incontestables de telles opérations au prisme des problèmes sociaux qui s'ensuivent, en s'appuyant sur le cas quiténien. Elle observe l'impact des phénomènes de « gentrification » et pose la question du droit des secteurs populaires à vivre dans la ville « revitalisée».

Une conférence finale referme le colloque en proposant un premier bilan des principaux traits des politiques culturelles de conservation patrimoniale en Équateur depuis 20075. Cette conférence de clôture aborde la question du rôle de la désormais historique Casa de la Cultura et observe qu'elle peine à se synchroniser avec les politiques énoncées par le Ministère de la Culture équatorienne depuis I'arrivée de Rafael Correa au pouvoir. Depuis 2008, I'institution est reléguée progressivement au rang d'entité conservatrice d'un patrimoine culturel national alors qu'elle avait l'ambition de devenir, dans le cadre de la « Révolution culturelle» souhaitée et annoncée par le ministère, un acteur à part entière dans une politique culturelle commune. Ce regard critique pose plus généralement la question de la « ciudadanización » des patrimoines, qui fait des citoyens les destinataires finaux de toute action publique de conservation patrimoniale.

David MACIAS, Alexis MEDINA, Emmanuelle SINARDET

5 Anne-Claudine Morel (Université de Nice), «La Casa de la Cultura : dépositaire d'un héritage culturel national ou bien acteur d'une politique culturelle innovante? ». 